\title{
Foreword: Materials for energy harvesting, conversion and storage (ICOME 2016)
}

The 2015 issue Materials for Energy Harvesting, Conversion and Storage [1] was the subject of a thematic opening on the coupling between materials and energy. We have largely emphasized the complementarity of a crossed view between mathematics and physics as well as the path towards applications of technological breakdown such as nanomaterials for energy [1-3]. It included work on phase change materials [5-7] photovoltaic materials [8-13] and plant composites (biosourced materials) [14]. This special edition is a continuation and is intended as a snapshot of some advances at the material/energy interface. It will also aim to stimulate the interest of young researchers in an investment from a multidisciplinary point of view in order to meet the new challenges presented by the open questions at the border of their own discipline.

This approach is illustrated here in particular for problems of anisothermal fluid physics, heat and mass transfer in porous media, biosourced materials and their thermo-physical properties, towards thermodynamic cycles for alternative energies, exchangers for power plant exchangers, the theme of solar and photovoltaics, etc. These are applications where physics will continue to play a leading role in understanding the phenomena and developing new solutions. These subjects inevitably integrate societal choices that are increasingly influenced by the participatory citizenship. It requires to be extended to the fields of energy mutation, materials, their natural and omnipresent coupling, Impact on its economy, health and environment. Energy strategies are becoming a major issue for established and transforming democracies and a determining factor in peace and social justice in the world.

Scientific methodology, increasingly benefiting from new algorithmic, numerical methods and material advances, is gaining relevance for experimentation and modeling in order to reach fine scales and unravel complex behaviors. These advances are sometimes made certain with a certain delay according to the disciplines. The introduction in this issue of the Lattice Boltzmann Method (LBM) [15] deserves a freeze. This is a different view on the method of solving matter conservation problems treated by coupled partial differential equations. LBM completes the MD method and offers an alternative way of solving problems in a physically accessible way, and in this respect arouses the interest of many researchers in digital physics, especially for complex geometric structures or with many degrees of freedom.

Mohammed El Ganaoui

Université de Lorraine, France

Jean-Michel Nunzi

Queens University, Kingston, ON, Canada

Rachid Bennacer

Université Paris Saclay, ENS Cachan, France 
L'édition 2015 de Materials for Energy Harvesting, Conversion and Storage [1] a été l'objet d'une ouverture thématique sur le couplage matériaux et énergie. Nous y avons largement mis en exergue la complémentarité d'un regard croisé entre les mathématiques et la physique ainsi que le chemin vers des applications de rupture technologique comme les nanomatériaux pour l'énergie [2-4]. Elle incluait ainsi des travaux traitant de matériaux à changement de phase [5-7] de matériaux photovoltaïques [8-13] et de composites végétaux (matériaux biosourcés) [14]. La présente édition spéciale s'inscrit dans la continuité et se veut une image instantanée de certaines avancées à l'interface matériaux/énergie. Elle visera également à stimuler l'intérêt des jeunes chercheurs pour un investissement tenant compte d'un point de vue pluridisciplinaire pour relever les nouveaux défis présentés par les questions ouvertes dans leur propre discipline.

Cette démarche est illustrée ici en particulier pour des problèmes de physique des fluides, des transferts de chaleur et de masse dans des milieux poreux, les matériaux biosourcés et leurs propriétés thermo physiques, vers les cycles thermodynamiques pour les énergies alternatives, les échangeurs pour centrales énergétiques, le thème du solaire et du photovoltaïque, etc. Autant d'applications où la physique continuera à jouer un rôle moteur pour parvenir à comprendre les phénomènes et à élaborer des solutions nouvelles. Ces sujets intègrent inéluctablement aujourd'hui des choix sociétaux de plus en plus influencés par une citoyenneté participative et exigeant qu'on l'étende dans les domaines des choix de l'énergie, des matériaux, de leur couplage naturel et omniprésent et de l'impact sur son économie, sa santé et son environnement. Les stratégies énergétiques deviennent un enjeu majeur des démocraties et un facteur déterminant de paix et de justice sociale dans le monde.

La méthodologie scientifique, bénéficiant de plus en plus des avancées algorithmiques et matérielles gagne en pertinence pour l'expérimentation et la modélisation afin d'atteindre des échelles fines et démêler des comportements complexes. Ces avancées sont des fois communiquées certain avec retard selon les disciplines. L'introduction dans ce numéro de la simulation par la méthode de Boltzmann sur réseau (LBM) [15] mérite un arrêt sur image. Il s'agit d'un regard différent sur la méthode de résolution des problèmes de bilan de conservation de matière traduite par des équations aux dérivées partielles couplées. La LBM offre une autre voie de résolution des problèmes de manière accessible au sens physique et suscite à ce propos l'intérêt de nombreux chercheurs en physique numérique, notamment pour les aspect géométriques complexes ou présentant de nombreux degrés de liberté.

\section{Mohammed El Ganaoui}

Université de Lorraine, France

Jean-Michel Nunzi

Queens University, Kingston, ON, Canada

Rachid Bennacer

Université Paris Saclay, ENS Cachan, France

\section{References}

1. R. Bennacer, M. El Ganaoui, J.M. Nunzi, Eur. Phys. J. Appl. Phys. 74, 24601 (2016)

2. A.B. Dalton, J.N. Coleman, M.I.H. Panhuis, et al., J. Photochem. Photobiol.: A Chem. 144, 31 (2001)

3. N. Kaur, M. Singh, D. Pathak, et al., Synth. Met 190, 20 (2014)

4. L. Sicot, C. Fiorini, A. Lorin, et al., Solar Energy Mater. Solar Cells 63, 49 (2000)

5. R. El Bahjaoui, H. El Qarnia, M. El Ganaoui, Eur. Phys. J. Appl. Phys. 74, 24616 (2016)

6. C. Bénard, R. Bénard, R. Bennacer, D. Gobin, Phys. Fluids 8, 112 (1996)

7. I. Hawachi, H. Sammouda, R. Bennacer, Int. J. Technology 2, 142 (2014)

8. N. Fellahi, M. Addou, A. Kachouane, M. El Jouad, Z. Sofiani, Eur. Phys. J. Appl. Phys. 74, 24611 (2016)

9. A. Bakour, M. Baitoul, E. Faulques, J. Wery-Venturini, Eur. Phys. J. Appl. Phys. 74, 24609 (2016)

10. M. Boutahir, A. Rahmani, H. Chadli, A. Rahmani, Eur. Phys. J. Appl. Phys. 74, 24605 (2016)

11. M. Hssein, L. Cattin, M. Morsli, M. Addou, J.-Ch. Bernède, Eur. Phys. J. Appl. Phys. 74, 24604 (2016)

12. Z. El Jouad, L. Cattin, F. Martinez, G. Neculqueo, G. Louarn, M. Addou, P. Predeep, J. Manuvel, J.-C. Bernède, Eur. Phys. J. Appl. Phys. 74, 24603 (2016)

13. O. Skhouni, A. El Manouni, B. Mari, H. Ullah, Eur. Phys. J. Appl. Phys. 74, 24602 (2016)

14. A. Zerriaa, M. El Ganaoui, C. Gerardin, A. Tazibt, S. Gabsi, Eur. Phys. J. Appl. Phys. 74, 24607 (2016)

15. A. Boutra, K. Ragui, R. Bennacer, Y.K. Benkahla, Eur. Phys. J. Appl. Phys. 74, 24612 (2016) 